Delft University of Technology

\title{
Torque Vectoring Control on Ice for Electric Vehicles with Individually Actuated Wheels
}

Agliullin, Timur; Ivanov, Valentin; Ricciardi, Vincenzo; Acosta, Manuel; Augsburg, Klaus; Sandu, Corina; Shyrokau, Barys; Savitski, Dzmitry

DOl

10.1007/978-3-030-38077-9_177

Publication date

2019

Document Version

Final published version

Published in

Advances in Dynamics of Vehicles on Roads and Tracks

\section{Citation (APA)}

Agliullin, T., Ivanov, V., Ricciardi, V., Acosta, M., Augsburg, K., Sandu, C., Shyrokau, B., \& Savitski, D. (2019). Torque Vectoring Control on Ice for Electric Vehicles with Individually Actuated Wheels. In M.

Klomp, F. Bruzelius, J. Nielsen, \& A. Hillemyr (Eds.), Advances in Dynamics of Vehicles on Roads and Tracks : Proceedings of the 26th Symposium of the International Association of Vehicle System Dynamics, IAVSD 2019 (pp. 1543-1551). (Lecture Notes in Mechanical Engineering). Springer. https://doi.org/10.1007/978-3-030-38077-9_177

Important note

To cite this publication, please use the final published version (if applicable).

Please check the document version above. 
Green Open Access added to TU Delft Institutional Repository

'You share, we take care!' - Taverne project

https://www.openaccess.nl/en/you-share-we-take-care

Otherwise as indicated in the copyright section: the publisher is the copyright holder of this work and the author uses the Dutch legislation to make this work public. 


\title{
Torque Vectoring Control on Ice for Electric Vehicles with Individually Actuated Wheels
}

\author{
Timur Agliullin ${ }^{1}$, Valentin Ivanov ${ }^{2(\bowtie)}, V^{(i n c e n z o ~ R i c c i a r d i ~}{ }^{2(凶)}$, \\ Manuel Acosta ${ }^{2}$, Klaus Augsburg ${ }^{2}$, Corina Sandu ${ }^{3}$, Barys Shyrokau ${ }^{4}$, \\ and Dzmitry Savitski ${ }^{2}$ \\ ${ }^{1}$ KNRTU-KAI, Kazan, Russia \\ 2 Technische Universität Ilmenau, Ilmenau, Germany \\ \{valentin.ivanov, vincenzo.ricciardi\}@tu-ilmenau.de \\ 3 Virginia Polytechnic Institute and State University, Blacksburg, USA \\ ${ }^{4}$ Delft University of Technology, Delft, The Netherlands
}

\begin{abstract}
Recent studies on torque vectoring control for electric vehicles proposed various efficient solutions demonstrating improvement of vehicle stability for evasive manoeuvres. However, the torque vectoring on very low friction surfaces such as black ice or wet snow is rarely investigated, especially for the electric vehicles with off-road capability. The presented study contributes to this topic by laying the groundwork for further advanced torque vectoring designs. Within the framework of this paper, the target vehicle is a sport utility vehicle equipped with four on-board electric motors controlling each wheel separately. The functionality of the developed controllers is tested under hardware-in-the-loop simulations for icy road conditions. For this purpose, the tyre model has been parameterized and validated based on the experimental data conducted on a unique terramechanics test rig at Virginia Polytechnic Institute and State University. The test results confirm very good functionality of the developed controllers and demonstrate an improvement of the electric vehicle driving performance.
\end{abstract}

Keywords: Torque vectoring $\cdot$ Electric vehicles $\cdot$ Yaw rate control

\section{Introduction}

Torque vectoring (TV) has been receiving an increasing attention in automotive research and development as an efficient extension of active safety systems combining functions of the vehicle stability control and driving experience enhancement. For the stability control, TV generates the vehicle yaw moment to correct excessive yaw rate or sideslip angle through redistribution of driving torques between individual wheels. Such torque-based stability control is relevant to all-wheel drive (AWD) vehicles with active inter-axle and inter-wheel differentials or to electric vehicles with individual inwheel or on-board motors. From analysis of known TV solutions, several control allocation criteria (Shimada et al. 1994; Yamakawa and Watanabe 2006) and various control architectures (Jalali et al. 2013; Bünte et al. 2014; Goggia et al. 2014; Lu et al. 2016) emerge from the literature. Available published results show that TV systems 
provide sufficient performance on high- and middle-friction surfaces, but the TV operation on very low-fiction surfaces has not been addressed sufficiently enough. The ice can be recognised as a case, where existing TV systems are approaching a limit in achievable improvement when conventional control methods are used, unless new disruptive control technologies are considered.

In the present work, the TV is achieved by applying the non-linear transient single-track model for yaw rate control. Several control methods are compared, i.e.: Proportional-Integral-Derivative (PID), Variable Structure Proportional-Integral (VSPI), First Order Sliding Mode (FOSM), Twisting Second-Order Sliding Mode (SOSM) and Integral Sliding Mode (ISM) control (Goggia et al. 2014). The functionality of the developed controllers is evaluated via hardware-in-the-loop (HIL) experiments with a real electronic control unit (ECU) interfaced with the vehicle dynamics simulator IPG CarMaker ${ }^{\circledR}$. An experimentally validated vehicle model is used during the simulations. The Magic Formula (MF) tyre model has been identified and validated against the experimental data obtained from the terramechanics test rig at Virginia Polytechnic Institute and State University, which allows for tyre testing on prepared real icy surfaces. The test rig is equipped with the Kistler wheel hub sensor to measure the forces and moments on the tested tyre with variation of tyre pressure, wheel loading, camber and toe angles. The next section will describe the architecture of the electric vehicle used in this study. Thereafter, results of a slowly increasing steer and a power oversteering manoeuvres on icy surface will be reported and analysed.

\section{Vehicle Model}

The adopted vehicle is based on the platform of the SUV Range Rover Evoque. The conventional powertrain has been replaced with four individual on-board electric motors, which allow for an individual control of each wheel (De Novellis et al. 2012). This configuration can realise the stabilizing yaw moment by means of torque vectoring. The vehicle is also equipped with on-board yaw rate and sideslip sensors. The main technical specifications of the modelled vehicle are listed in Table 1.

Table 1. Technical specifications of the vehicle model

\begin{tabular}{l|l}
\hline Specification & Quantity \\
\hline Mass & $2105 \mathrm{~kg}$ \\
\hline Yaw moment of inertia & $2760 \mathrm{~kg} \mathrm{~m}{ }^{2}$ \\
\hline Front/rear semi-wheelbase & $1.07 \mathrm{~m} / 1.59 \mathrm{~m}$ \\
\hline Front/rear track width & $1.625 \mathrm{~m} / 1.625 \mathrm{~m}$ \\
\hline Height of CoG & $0.66 \mathrm{~m}$ \\
\hline Driveline type & Individual on-board electric motors \\
\hline Motor type & $4 \times$ Switched Reluctance $($ SR) motors \\
\hline Gear ratio & 10.56 \\
\hline Nominal torque/power & $80 \mathrm{Nm} / 35 \mathrm{~kW}$ \\
\hline Peak torque/power & $200 \mathrm{Nm} / 75 \mathrm{~kW}$ \\
\hline Tyre size & $235 / 55 \mathrm{R} 19$ \\
\hline
\end{tabular}


As was mentioned before, the tyre model is based on Magic Formula (MF) using the parameterised data obtained from the terramechanic test rig at Virginia Polytechnic Institute and State University (Bhoopalam et al. 2015). The longitudinal slip of the tyre was controlled while the rig moved over the ice. The testing equipment measured longitudinal and normal forces as well as the absolute angles. Furthermore, the lateral tyre behavior was extracted from another set of experimental data and the coefficient were adjusted to align the lateral grip with the maximum longitudinal grip.

\section{Torque Vectoring System}

The developed TV system comprises control of vehicle yaw rate and side slip angle. The yaw rate controller is based on tracking of the yaw rate reference, which is derived from the non-linear transient single-track vehicle model, whereby an appropriate understeer gradient is selected. The sideslip angle controller uses the $\beta-\dot{\beta}$ phase plane trajectories in order to define the corresponding control error. The scheme of the TV control system is provided in Fig. 1. The yaw moment demand signals $\Delta M_{z, \dot{\psi}}$ and $\Delta M_{z, \beta}$ from both yaw rate and the sideslip angle controllers are inputs to the Control Allocation, which distributes the requested control demands to the wheel forces. The four wheels are driven by individual electric motors.

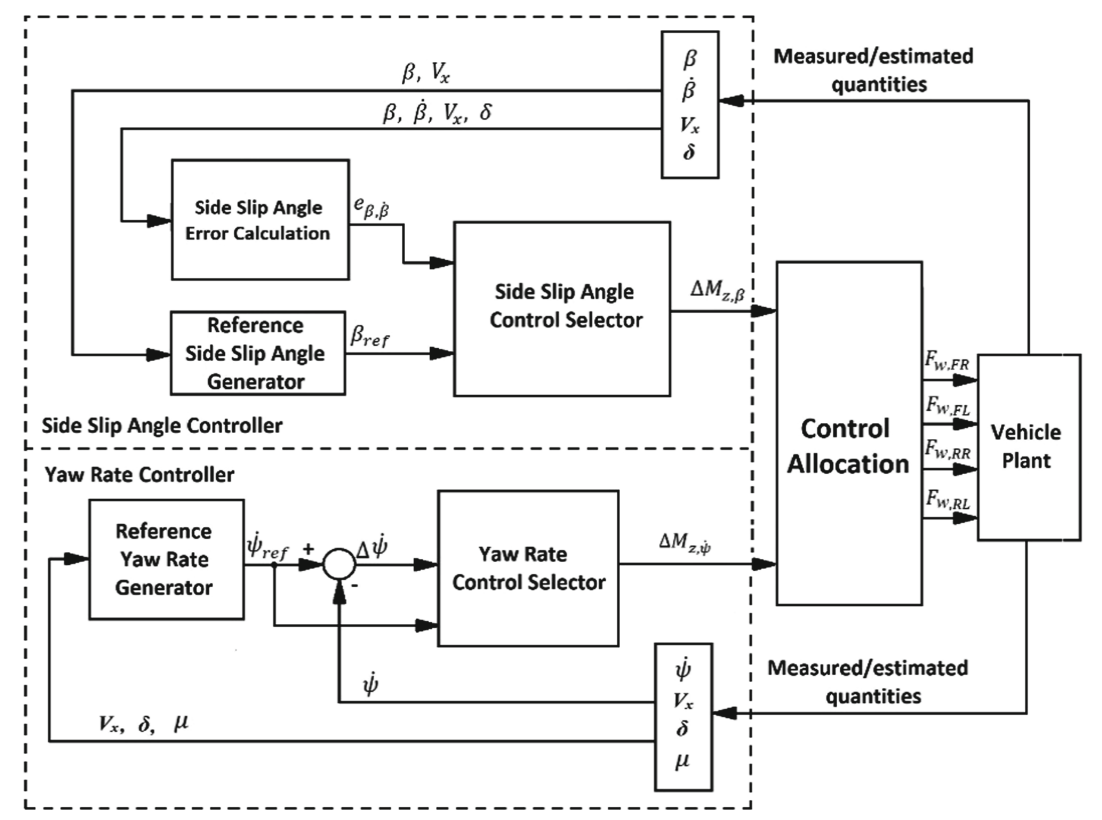

Fig. 1. Scheme of the torque vectoring control system. 


\subsection{Yaw Rate Control}

The reference value of vehicle yaw rate is calculated in the Reference Yaw Rate Generator (Fig. 1) as the function of the steering angle $\delta_{f}$ and the longitudinal velocity $V_{x}$ according to (Galip Ulsoy et al. 2012):

$$
\dot{\psi}_{r e f}=\frac{\delta_{f} V_{x}}{L+K_{u s} V_{x}^{2}},
$$

where $L$ is the wheel base $(\mathrm{m}), K_{u s}$ is the vehicle understeer gradient $\left(\operatorname{rad} /\left(\mathrm{m} / \mathrm{s}^{2}\right)\right)$. The reference yaw rate is saturated by the maximum achievable value of yaw rate $\dot{\psi}_{\text {lim }}$ for the given tyre-road friction coefficient $\mu$ and the vehicle velocity $V_{x}$, as follows (Van Zanten 2002):

$$
\left|\dot{\psi}_{\text {lim }}\right| \leq\left|\mu g / V_{x}\right|
$$

\subsection{Sideslip Angle Control}

The lateral vehicle stability must be also ensured with respect to the sideslip angle. Several phase portraits of the vehicle dynamics were obtained offline for different steering angles and longitudinal velocities using the nonlinear single-track model. The stable regions on the $\beta-\dot{\beta}$ phase plane were defined by the four points on the outermost phase trajectories meeting in the equilibrium point: two saddle points ( 1 and 2 in Fig. 2) and two limiting points (3 and 4 in Fig. 2). The coordinates of these points are recorded and stored in the lookup tables of the Side Slip Angle Error Calculation block (Fig. 1).

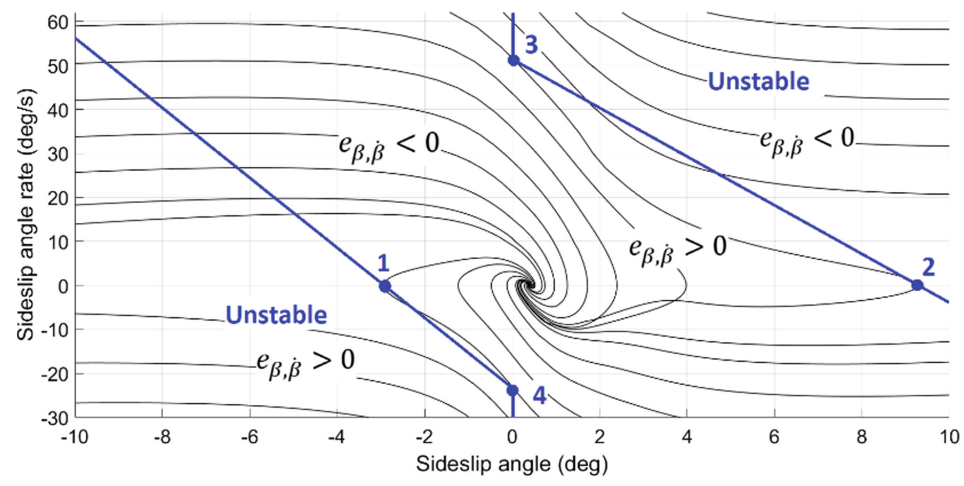

Fig. 2. Phase plane analysis of the vehicle side slip.

The combined control error of sideslip angle and its derivative $e_{\beta, \dot{\beta}}$ is calculated as the distance between the vehicle state point and the straight line defined by two points (either 1 and 4, or 2 and 3). The controller is activated or deactivated depending on the 
sign of $e_{\beta, \dot{\beta}}$, as shown in Fig. 2, so that the control action $\Delta M_{z, \beta}$ is generated only when the vehicle state is located in the unstable regions of the phase plane.

\subsection{Control Performance Evaluation}

To objectively evaluate the performance of each control strategy, the current work introduces the weighted control performance index (WCPI). The WCPI is a dimensionless quantity, where lower values indicate better control performance. The WCPI is formulated as follows:

$$
W C P I=w_{1} \frac{R M S E_{\dot{\psi}}}{R M S E_{\dot{\psi}, \text { in }}}+w_{2} \frac{R M S E_{\beta}}{R M S E_{\beta, \text { in }}}+w_{3} \frac{I T A E_{\dot{\psi}}}{I T A E_{\dot{\psi}, \text { in }}}+w_{4} \frac{I T A E_{\beta}}{I T A E_{\beta, \text { in }}}+w_{5} \frac{I A C A}{M_{z, M} t_{m}}+w_{6} \frac{V_{x, f i n}}{V_{x, \text { in }}}
$$

$R M S E_{\dot{\psi}}$ and $R M S E_{\beta}$ are the root-mean-squares of error for the yaw rate and the side slip control, respectively; $I T A E_{\dot{\psi}}$ and $I T A E_{\beta}$ are integrals of the time-weighted absolute value of the error. $R M S E_{\dot{\psi}, \text { in }}, R M S E_{\beta, i n}, I T A E_{\dot{\psi}, i n}$, and $I T A E_{\beta, \text { in }}$ are the values of the corresponding parameters for the baseline vehicle (i.e. without TV); IACA is integral of the absolute value of control action; $M_{z, M}$ is the maximum achievable yaw moment generated by the TV system; $t_{m}$ is the duration of the manoeuvre; $V_{x, i n}$ and $V_{x, \text { fin }}$ are the vehicle longitudinal velocities at the beginning and at the end of the maneuver, respectively. The values of weights $w_{1}, w_{2}, w_{3}$, and $w_{4}$ were chosen according to the following conditions: (1) the sum of the weights should be equal to 1; (2) all the components in (3) should have the same order of magnitude; (3) the priority was given to the error minimisation criteria, i.e. the RMSE and ITAE components have higher weights than the others.

\section{Simulation Results}

The functionality of the developed controllers is tested under HIL environment consisting of an ECU integrated with the experimentally validated model of a full electric SUV in IPG CarMaker ${ }^{\circledR}$. A fixed time step of $1 \mathrm{~ms}$ was set during the simulations and an additive noise model was employed to incorporate white Gaussian noise into the simulation signals.

In order to test the effectiveness of the developed controllers in steady-state conditions, a slowly increasing steer manoeuvre was performed. The longitudinal velocity was set equal to $30 \mathrm{~km} / \mathrm{h}$, and the steering wheel angle was increasing at the rate of $13.5 \%$. The yaw rate characteristics for all the controllers and the vehicle without TV are represented in the Fig. 3. The values of the weighted control performance index calculated using the expression (3) for all control methods are reported beside Fig. 3 . The overall performance of all controllers is illustrated in the form of spider diagram in Fig. 4. The sideslip angle control was not activated during this manoeuvre since the vehicle state on the sideslip phase plane did not exceed the stability region defined according to Chapter 3 of the paper. 


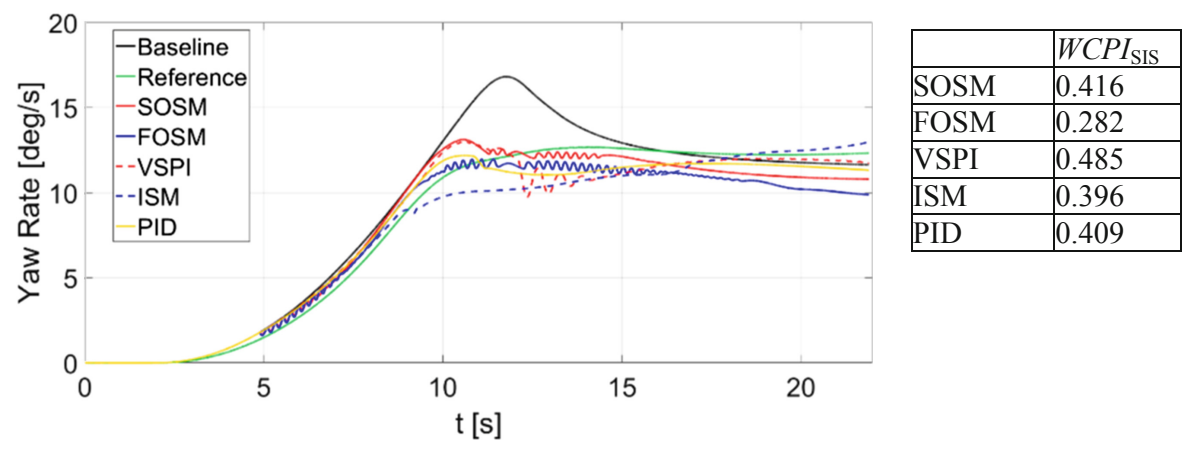

Fig. 3. Yaw rate diagrams and WCPI values for a slowly increasing steer test.

The yaw rate of baseline vehicle without TV significantly deviates from the reference value. All control strategies implemented in the TV system demonstrated considerable improvement in tracking of the reference yaw rate. The FOSM control received the best WCPI due to the lowest RMSE and ITAE values, but it provided the highest $I A C A$, due to the oscillating nature of its control action.

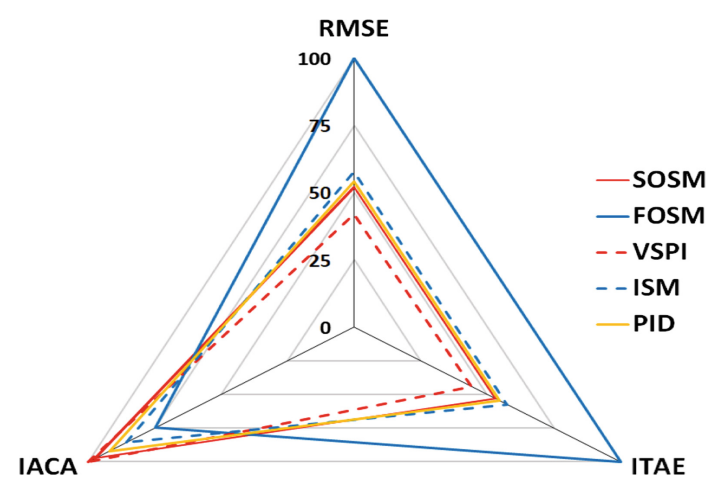

Fig. 4. Spider plot for the slowly increasing steer test.

The control performance in transient conditions was evaluated using the power oversteering manoeuvre. The simulation was performed at constant steering angle of $30^{\circ}$ and initial speed of $10 \mathrm{~km} / \mathrm{h}$. After $2 \mathrm{~s}$, the virtual driver applies traction torque to increase the speed up to $30 \mathrm{~km} / \mathrm{h}$. As in the previous case, the yaw rate for the baseline vehicle and the vehicle with TV are shown in Fig. 5 along with the reference value. The WCPI indexes are listed in Fig. 5. In addition, the side slip phase trajectories are presented in Fig. 6, while the spider diagram is shown in Fig. 7. 


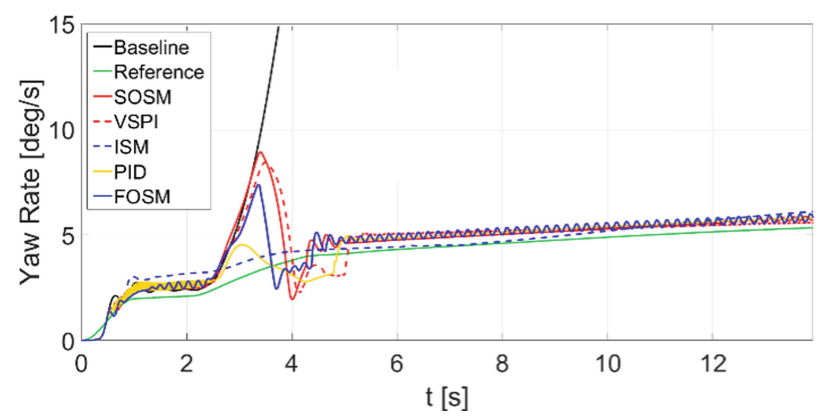

\begin{tabular}{|l|l|}
\hline & $W C P I_{\text {POS }}$ \\
\hline SOSM & 0.114 \\
\hline FOSM & 0.118 \\
\hline VSPI & 0.079 \\
\hline ISM & 0.108 \\
\hline PID & 0.113 \\
\hline
\end{tabular}

Fig. 5. Yaw rate diagrams and WCPI values for a power oversteering test.

The baseline vehicle exhibits an oversteering behaviour, resulting in a loss of control and vehicle spin-out. This behaviour is illustrated in Fig. 6, where the phase trajectory of the baseline vehicle exceeds the stability boundary in the third quadrant of the phase plane. As can be noted, all control strategies keep the vehicle state in a stable region; therefore, the sideslip controller was not activated during the manoeuvre.

As per Fig. 7, the control methods received similar values of WCPI. However, the best results were demonstrated by the VSPI controller mostly due to the significantly lower IACA value in comparison with other control strategies. The ISM controller provided the best performance of the yaw rate tracking in these conditions, while the sliding mode controllers allowed considerable deviation of the yaw rate from the reference at the beginning of vehicle acceleration.

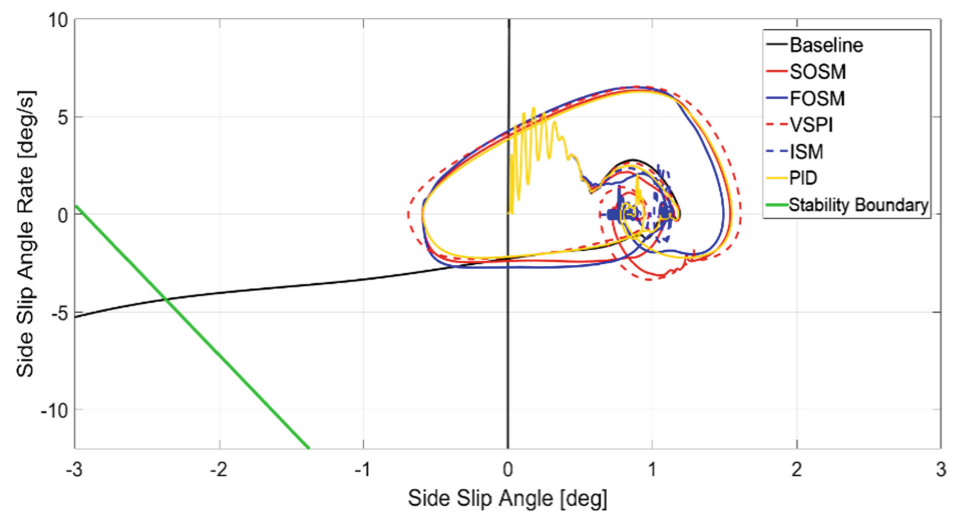

Fig. 6. Side slip phase trajectories for the power oversteering test. 


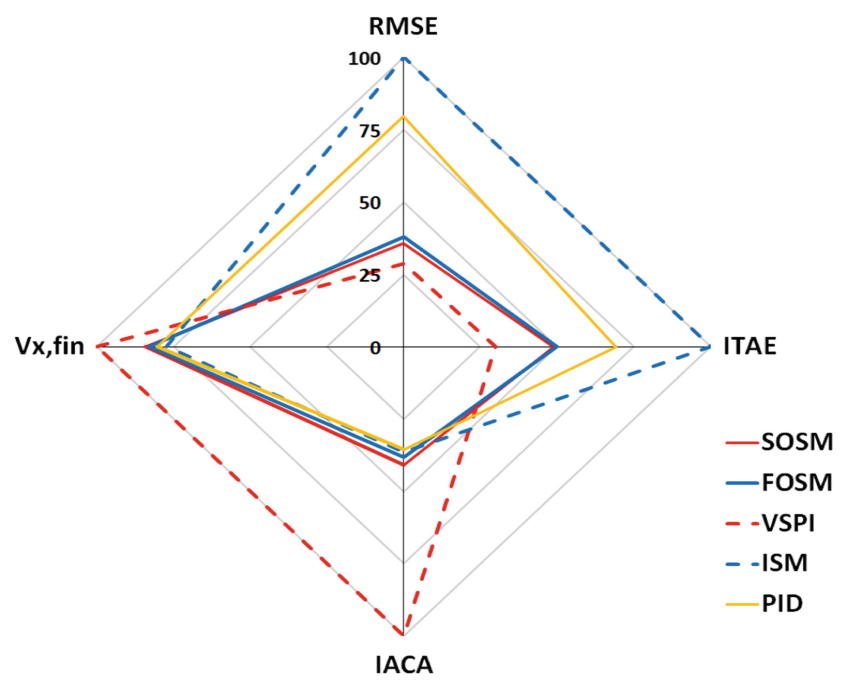

Fig. 7. Spider plot for the power oversteering test.

\section{Conclusions}

The proposed work addresses the performance of five control methods implemented for torque-vectoring control of an electric vehicle with individual on-board motors. The functionality of the developed controllers is tested under HIL environment consisting of an ECU integrated with the experimentally validated model of a full electric SUV in IPG CarMaker ${ }^{\circledR}$. The simulation was conducted on a very low-friction surface corresponding to icy road conditions. Particularly, the tyre model was parametrised against experimental tests on icy surface using the terramechanics test rig at Virginia Polytechnic Institute and State University. To evaluate the controllers' performance, the weighted control performance index $(W C P I)$ is considered. The WCPI takes into account the tracking accuracy of reference yaw rate, the quality of transient process and sideslip angle error minimisation, the extent of control action (i.e. its efficiency), as well as longitudinal velocity variations resulting from the TV system operation. The implemented control strategies demonstrated sufficient performance, significantly improving the values of all objective evaluation criteria. The best WCPI values were achieved by the FOSM controller in steady-state conditions, and by the VSPI controller in the transient manoeuvres.

\section{References}

Bhoopalam, A., Sandu, C., Taheri, S.: Experimental investigation of pneumatic tire performance on ice: Part 1 - Indoor study. J. Terrramech. 60, 43-54 (2015)

Bünte, T., Kaspar, S., Hohmann, S., Brembeck, J.: Inverse model based torque vectoring control for a rear wheel driven battery for electric vehicle. In: The 19th IFAC World Congress, Cape Town, South Africa (2014) 
De Novellis, L., Sorniotti, A., Gruber, P., Shead, L., Ivanov, V., Hoepping, K.: Torque vectoring for electric vehicles with individually controlled motors: state-of-the-art and future developments. World Electr. Veh. J. 5(2), 617-628 (2012)

Galip Ulsoy, A., Peng, H., Çakmakci, M.: Automotive Control Systems. Cambridge University Press, Cambridge (2012). ISBN 978-1-107-01011-6

Goggia, T., Sorniotti, A., De Novellis, L., Ferrara, A.: Torque-vectoring control in fully electric vehicles via integral sliding modes, In: American Control Conference (ACC), Portland, OR, USA (2014)

Jalali, K., Uchida, T., Lambert, S., McPhee, J.: Development of an advanced torque vectoring control system for an electric vehicle with in-wheel motors using soft computing techniques. SAE Int. J. Altern. Powertrains 2(2), 261-278 (2013)

Lu, Q., Sorniotti, A., Gruber, P., Teunissen, J., De Smet, J.: Ho loop shaping for the torquevectoring control of electric vehicles: Theoretical design and experimental as-sessment. Mechatronics 35, 32-43 (2016)

Shimada, K., Shibahata, Y.: Comparison of three active chassis control methods for stabilizing yaw moments. SAE Technical Paper Series, no. 940870 (1994)

Van Zanten, A.T.: Evolution of electronic control systems for improving the vehicle dynamic behavior. In: Proceedings of the International Symposium on Advanced Vehicle Control (2002)

Yamakawa, J., Watanabe, K.: A method of optimal wheel torque determination for independent wheel drive vehicles. J. Terrramech. 43(3), 269-285 (2006) 